\title{
FINDING EXPECTED REVENUES IN G-NETWORK WITH SIGNALS AND CUSTOMERS BATCH REMOVAL
}

\author{
Mikhail MatalytSKI and DMitRy Kopats \\ Faculty of Mathematics and Computer Science, \\ Grodno State University, \\ Grodno, Belarus \\ E-mail: m.matalytski@gmail.com
}

\begin{abstract}
The paper provides an analysis of G-network with positive customers and signals when signals arriving to the system move customer to another system or destroy in it a group of customers, reducing their number to a random value that is given by a probability distribution. The signal arriving to the system, in which there are no positive customers, does not exert any influence on the queueing network and immediately disappears from it. Streams of positive customers and signals arriving to each of the network systems are independent. Customer in the transition from one system to another brings the latest some revenue, and the revenue of the first system is reduced by this amount. A method of finding the expected revenues of the systems of such a network has been proposed. The case when the revenues from transitions between network states are deterministic functions depending on its states has been considered. A description of the network is given, all possible transitions between network states, transition probabilities, and revenues from state transitions are indicated. A system of difference-differential equations for the expected revenues of network systems has been obtained. To solve it, we propose a method of successive approximations, combined with the method of series. It is proved that successive approximations converge to the stationary solution of such a system of equations, and the sequence of approximations converges to a unique solution of the system. Each approximation can be represented as a convergent power series with an infinite radius of convergence, the coefficients of which are related by recurrence relations. Therefore, it is convenient to use them for calculations on a PC. The obtained results can be applied in forecasting losses in information and telecommunication systems and networks from the penetration of computer viruses into it and conducting computer attacks.
\end{abstract}

Keywords: applied probability, queueing theory

\section{INTRODUCTION}

A survey of the results obtained for the queueing systems (QSs) and networks in the stationary regime can be found in [1]. The queueing networks with revenues in the non-stationary regime were considered in [2-5]. The customer passing from one QS to another brings to the latter some revenue, and the revenue of the former QS is decreased by the same value. At 
that, the revenues from the transitions between the network states depended on their states and time or were random variable with given moments of the first and second orders. The review paper [2] present the results of studies, analysis, optimization, and selection of the optimal strategies in the Markov networks with revenues, describe various their applications as the probabilistic models for forecasting the anticipated revenues in the information telecommunication systems and networks, where, for example, customer servicing on a server brings an revenue to the servicer, as well as in the insurance companies, logistic transport systems, production systems, and other objects.

G-networks have been used to control packet networks in real time [6] and the transient behavior of the G-network as a control element has an impact on the behavior of the packet network itself and therefore is of practical value. Furthermore it is interesting to note that for certain QSs [7] the stationary probabilities do not depend on the inter-arrival and service time distributions [7], while we would not expect this to hold for transients in general. Finally, certain generalizations of G-networks [8] do not have any impact on the stationary probability distributions [8], while intuitively we would expect that the transients will be affected.

\section{NETWORK DESCRIPTION. THE PROBLEM}

We will consider an open G-network [9] with $n$ single-queue QSs. Simple flow of customers arrives to the $S_{i}$ system from external environment (QS $S_{0}$ ) with the rate $\lambda_{0 i}^{+}$, additional simplest signal flow also arrives with the rate $\lambda_{0 i}^{(1)}, i=\overline{1, n}$. Assume all customer flows arriving to QS are independent. The service durations of positive customers in $i$-th QS distributed exponentially with the rate $\mu_{i}, i=\overline{1, n}$. The positive customer processed by $S_{i}$ moves to QS $S_{j}$ as a positive customer with probability $p_{i j}^{+}$, moves as a negative customer with probability $p_{i j}^{-}$, and moves out of the network to the external environment with probability $p_{i 0}=1-\sum_{j=1}^{n}\left(p_{i j}^{+}+p_{i j}^{-}\right), i, j=\overline{1, n}$.

Let at some time moment there are $k_{i} \geq B_{i}$ positive customers in $i$ th QS, where $B_{i}-$ random integer value. When signal arrives in the system, acting as a negative customer in this system, the number of positive customers therein is reduced by $B_{i}$ ( $B_{i}$ positive customers are removed immediately). If $k_{i}<B_{i}$ then $i$ th QS does not remain customers. Random value $B_{i}$ determines the maximum size of the customers removal batch at QS $S_{i}$, and has an arbitrary discrete distribution law: $P\left\{B_{i}=m\right\}=\pi_{i m}, m \geq 1, E\left\{B_{i}\right\}=b_{i}$.

The signal [10], coming in QS $S_{i}$ with no positive customers, does not have any impact on the network and immediately disappears from it. Otherwise, if the system $S_{i}$ is not empty and a signal arrives into it, there can occur following events: incoming signal instantly moves the positive customer from the system $S_{i}$ into the system $S_{j}$ with probability $q_{i j}$, in this case, signal is referred to as a trigger; signal acts as a negative customer and destroys a group of positive customers in QS $S_{i}$ with probability $q_{i 0}=1-\sum_{j=1}^{n} q_{i j}$ [11].

The network state at time $t$ is described by the vector $(\vec{k}, t)=\left(k_{1}, k_{2}, \ldots, k_{n}, t\right)$, which forms a homogeneous Markov process with a countable number of states, that means there are $k_{i}$ positive customers in QS $S_{i}$ at time $t, i=\overline{1, n}$.

Customers during the transition from one to another QS bring the latest some revenue, and the revenue of the first QS is reduced by this amount. Revenue generally can take both positive and negative values (losses). Revenues of the G-network with the group removal of positive customers, but without signals were considered in [12].

It is required to find the average revenue that customers bring to $i$ th QS during time $t, i=\overline{1, n}$. It should be noted that the method of finding the expected revenues in various 
queueing networks of small dimension, when the revenues from the transitions between their states depend on these states and time, and also the approximate method of finding them in the case when the revenues from the transitions are random variables, are described in the articles [2-5]. Here we shall use a modified method of successive approximations applied to G-networks in $[13,14]$.

\section{ABOUT FINDING EXPECTED REVENUES OF THE NETWORK SYSTEMS}

We introduce some notation. Let $I_{i}$ - zero vector of dimension $n$, consisting of zeros, with the exception of the component with number $i$, which is equal to 1 ; let $v_{i}(\vec{k}, t)$ be the expected revenue obtained by the $i$ th QS in time $t$, if at the initial time instant the network is in the state $\vec{k} ; u(x)$ - unit Heaviside function, $u(x)=\left\{\begin{array}{l}1, x>0, \\ 0, x \leq 0,\end{array}\right.$. The following transitions of the Markov random process to the state $(\vec{k}, t)$ during the time $\Delta t$ are possible:

(1) From the state $\left(\vec{k}-I_{j}, t\right), j \neq i$, in this case in the $j$ th QS in time $\Delta t$ a positive customer will arrive with a probability $\lambda_{0 j}^{+} u\left(k_{j}\right) \Delta t+o(\Delta t), i=\overline{1, n}$, the revenue of the system $S_{i}$ in this case will be $r_{i}(\vec{k}) \Delta t+v_{i}\left(\vec{k}-I_{j}, t\right)$; if $i=j$ then the revenue of the system $S_{i}$ will be $r_{0 i}\left(\vec{k}-I_{i}, t\right)+v_{i}\left(\vec{k}-I_{i}, t\right)$, where $r_{0 i}\left(\vec{k}-I_{i}, t\right)$ the revenue of the $i$ th system from the given transition.

(2) From the state $\left(\vec{k}+I_{j}, t\right), j \neq i$, wherein the positive customer leaves the network to the external environment or moves to the $j$ th QS as a signal if there were no customers in it; the probability of such an event is equal to $\left(\mu_{i} p_{i 0}+\right.$ $\left.\mu_{i} p_{i j}^{-}\left(1-u\left(k_{j}\right)\right)\right) \Delta t+o(\Delta t), i, j=\overline{1, n}$, the revenue of the system $S_{i}$ in this case will be $r_{i}(\vec{k}) \Delta t+v_{i}\left(\vec{k}+I_{j}, t\right)$; if $i=j$ then the revenue of the system $S_{i}$ will be $-R_{i 0}\left(\vec{k}+I_{i}, t\right)+v_{i}\left(\vec{k}+I_{i}, t\right)$, where $R_{i 0}\left(\vec{k}+I_{i}, t\right)$ the revenue of the $i$ th system from the given transition.

(3) From the state $\left(\vec{k}+I_{j}-I_{s}, t\right), j, s \neq i$, in this case, after the end of the service of the positive customer in the $j$ th QS it is moved to the $s$ th QS again as a positive customer or arrived to the $i$ th QS signal instantly moves the positive customer from the $i$ th QS to the $j$ th QS; the probability of this event is equal to $\left(\mu_{j} p_{j s}^{+}+\lambda_{0 j}^{(c)} q_{j s}\right) u\left(k_{s}\right)$ $\Delta t+o(\Delta t), i, j=\overline{1, n}$, the revenue of the system $S_{i}$ in this case will be $r_{i}\left(\vec{k}+I_{j}-\right.$ $\left.I_{s}\right) \Delta t+v_{i}\left(\vec{k}+I_{j}-I_{s}, t\right)$; if $j=i$ then $-r_{i s}\left(\vec{k}+I_{i}-I_{s}, t\right)+v_{i}\left(\vec{k}+I_{i}-I_{s}, t\right)$.

(4) From the state $\left(\vec{k}+I_{j}+I_{s}-I_{c}, t\right), c, s, j \neq i$, in this case, after the end of the service of the customer in the $j$ th QS, it is sent to the sth QS a signal, which instantly moves the positive customer from the $j$ th QS with a number $c$; the probability of this event is equal to $\mu_{j} p_{j s}^{-} q_{s c} u\left(k_{c}\right) \Delta t+o(\Delta t), \quad i, j, s=\overline{1, n}$, the revenue of the system $S_{i}$ in this case will be $r_{i}\left(\vec{k}+I_{j}+I_{s}-I_{c}, t\right) \Delta t+v_{i}\left(\vec{k}+I_{j}+I_{s}-I_{c}, t\right)$; if $j=i$ the revenue will be $-r_{j s c}\left(\vec{k}+I_{j}+I_{s}-I_{c}, t\right)+v_{i}\left(\vec{k}+I_{j}+I_{s}-I_{c}, t\right)$, and if $s=i$ it will be equal to $-r_{j i c}\left(\vec{k}+I_{j}+I_{i}-I_{c}, t\right)+v_{i}\left(\vec{k}+I_{j}+I_{i}-I_{c}, t\right)$, otherwise $r_{j s i}\left(\vec{k}+I_{j}+I_{s}-I_{i}, t\right)+v_{i}\left(\vec{k}+I_{j}+I_{s}-I_{i}, t\right)$.

(5) From the state $\left(\vec{k}+m I_{j}, t\right), j \neq i$, in this case, the signal from the outside arrives to the $j$ th QS and destroys in it a group of positive customers value of $m$; the probability of such an event is equal to $\lambda_{0 j}^{(c)} q_{j 0} \sum_{m=1}^{\infty} \pi_{j m} \Delta t+o(\Delta t), \quad i=\overline{1, n}$; in this case the revenue of the $S_{i}$ will be $r_{i}\left(\vec{k}+m I_{j}, t\right) \Delta t+v_{i}\left(\vec{k}+m I_{j}, t\right)$; otherwise $-R_{i}\left(\vec{k}+m I_{i}, t\right)+v_{i}\left(\vec{k}+m I_{i}, t\right)$. 
(6) If the signal arrives from outside to the $j$ th QS, $j \neq i$, in which there are no customers, it can wait for any group of customers and destroy it; the probability of such an event is equal to $\lambda_{0 j}^{(c)} q_{j 0}\left(1-u\left(k_{j}\right)\right) \sum_{m=1}^{\infty} \pi_{j m} \sum_{r=1}^{m-1} P\left(\vec{k}+r I_{j}, t\right) \Delta t+o(\Delta t), i, j=\overline{1, n}$, in this case the revenue of the $S_{i}$ will be $\sum_{r=1}^{m-1} r_{i}\left(\vec{k}+r I_{j}, t\right) \Delta t+v_{i}\left(\vec{k}+r I_{j}, t\right)$; if $i=j$ then $-\sum_{r=1}^{m-1} R_{i}\left(\vec{k}+r I_{i}, t\right) \Delta t+v_{i}\left(\vec{k}+r I_{i}, t\right)$.

(7) From the state $\left(\vec{k}+I_{j}+m I_{s}, t\right), j, s \neq i$, in this case, after the end of the customer service in the $j$ th QS, it is sent to the $s$ th QS as a signal that destroys a random group of positive customers; the probability of such an event is equal to $\mu_{j} p_{j s}^{-} q_{s 0} \sum_{m=1}^{\infty} \pi_{s m} \Delta t+o(\Delta t), i, j, s=\overline{1, n}$; the revenue of the system $S_{i}$ in this case will be $r_{i}\left(\vec{k}+I_{j}+m I_{s}, t\right) \Delta t+v_{i}\left(\vec{k}+I_{j}+m I_{s}, t\right)$; if $i=j$, it will be equal to $r_{i s}\left(\vec{k}+I_{i}+m I_{s}, t\right)+v_{i}\left(\vec{k}+I_{i}+m I_{s}, t\right)$; and if $s=i$, then $-R_{j i}\left(\vec{k}+I_{j}+m I_{i}, t\right)+$ $v_{i}\left(\vec{k}+I_{j}+m I_{i}, t\right)$.

(8) If after finishing the service of the customer in the $j$ th QS, it is sent to the sth QS as a signal that does not occur a customers in this QS, it waits for any number of customers and destroys them; the probability of such an event is equal to $\mu_{j} p_{j s}^{-}\left(1-u\left(k_{s}\right)\right) q_{s 0} \sum_{m=1}^{\infty} \pi_{s m} \sum_{r=1}^{m-1} P\left(\vec{k}+I_{j}+r I_{s}, t\right) \Delta t+$ $o(\Delta t), i, j, s=\overline{1, n}$; the revenue of the system $S_{i}$ in this case will be at $j=i \sum_{r=0}^{m-1}\left[r_{i s}\left(\vec{k}+I_{i}+r I_{s}, t\right)+v_{i}\left(\vec{k}+I_{i}+r I_{s}, t\right)\right] ;$ an if $s=i$ then $-\sum_{r=0}^{m-1}\left[R_{j i}(\vec{k}+\right.$ $\left.\left.I_{j}+r I_{i}, t\right)+v_{i}\left(\vec{k}+I_{j}+r I_{i}, t\right)\right]$.

(9) From the state $(\vec{k}, t)$, at the same time, no positive customers or signals are received in each, and no positive customer has been served in them for a time; the probability of this event is $1-\sum_{j=1}^{n}\left[\lambda_{0 j}^{+}+\left(\lambda_{0 j}^{(c)}+\mu_{j}\right) u\left(k_{j}\right)\right] \Delta t+o(\Delta t)$; the revenue of the system $S_{i}$ in this case will be $r_{i}(\vec{k}) \Delta t+v_{i}(k, t)$.

(10) From the remaining states with probability $o(\Delta t)$.

Using the total probability formula for the expectation, we obtain system of differencedifferential equations for revenue: $v_{i}(k, t)$ :

$$
\begin{aligned}
\frac{d v_{i}(\vec{k}, t)}{d t}= & r_{i}(\vec{k})-\sum_{j=1}^{n}\left[\lambda_{0 j}^{+}+\left(\lambda_{0 j}^{(c)}+\mu_{j}\right) u\left(k_{j}\right)\right] v_{i}(\vec{k}, t) \\
& +\sum_{\substack{j, s=1 \\
j, s \neq i}}^{n}\left\{\left(\mu_{j} p_{j 0}+\mu_{j} p_{j s}^{-}\left(1-u\left(k_{s}\right)\right)\right) v_{i}\left(\vec{k}+I_{j}, t\right)\right. \\
& +\lambda_{0 j}^{+} u\left(k_{j}\right) v_{i}\left(\vec{k}-I_{j}, t\right)+\lambda_{0 j}^{(c)} q_{j 0} \sum_{m=1}^{\infty} \pi_{j m} v_{i}\left(\vec{k}+m I_{j}, t\right) \\
& +\mu_{j} p_{j s}^{-} q_{j 0} \sum_{m=1}^{\infty} \pi_{s m} v_{i}\left(\vec{k}+I_{j}+m I_{s}, t\right) \\
& +\sum_{c=1}^{n} \mu_{j} p_{j s}^{-} q_{s c} u\left(k_{c}\right) v_{i}\left(\vec{k}+I_{j}+I_{s}-I_{c}, t\right) \\
& +\mu_{j} p_{j s}^{-}\left(1-u\left(k_{s}\right)\right) q_{s 0} \sum_{m=1}^{\infty} \pi_{s m} \sum_{r=1}^{m-1} v_{i}\left(\vec{k}+I_{j}+r I_{s}, t\right)
\end{aligned}
$$




$$
\begin{aligned}
& +\lambda_{0 j}^{(1)} q_{j 0}\left(1-u\left(k_{j}\right)\right) \sum_{m=1}^{\infty} \pi_{j m} \sum_{r=1}^{m-1} v_{i}\left(\vec{k}+r I_{j}, t\right) \\
& +\left(\lambda_{0 j}^{(c)} q_{j s}+\mu_{i} p_{j s}^{+} u\left(k_{s}\right)\right) v_{i}\left(\vec{k}+I_{j}-I_{s}, t\right) \\
& +\lambda_{0 i}^{+} u\left(k_{i}\right) v_{i}\left(\vec{k}-I_{i}, t\right)+\lambda_{0 i}^{(c)} q_{i 0} \sum_{m=1}^{\infty} \pi_{i m} v_{i}\left(\vec{k}+m I_{i}, t\right) \\
& +\mu_{i} p_{i s}^{-} q_{i s} \sum_{m=1}^{\infty} \pi_{s m} v_{i}\left(\vec{k}+I_{i}+m I_{s}, t\right) \\
& +\sum_{c=1}^{n} \mu_{i} p_{i s}^{-} q_{s c} u\left(k_{c}\right) v_{i}\left(\vec{k}+I_{i}+I_{s}-I_{c}, t\right) \\
& \left.+\mu_{i} p_{i s}^{-}\left(1-u\left(k_{s}\right)\right) q_{s 0} \sum_{m=1}^{\infty} \pi_{s m} \sum_{r=0}^{m-1} v_{i}\left(\vec{k}+I_{i}+r I_{s}, t\right)\right\} \\
& +\lambda_{0 i}^{(c)} q_{i 0}\left(1-u\left(k_{i}\right)\right) \sum_{m=1}^{\infty} \pi_{i m} \sum_{r=1}^{m-1} v_{i}\left(\vec{k}+r I_{i}, t\right) \\
& +\sum_{s=1}^{n}\left\{\left(\mu_{j} p_{j 0}+\mu_{j} p_{j s}^{-}\left(1-u\left(k_{s}\right)\right)\right) v_{i}\left(\vec{k}+I_{i}, t\right)\right. \\
& +\left(\lambda_{0 i}^{(c)} q_{i s}+\mu_{i} p_{i s}^{+} u\left(k_{s}\right)\right) v_{i}\left(\vec{k}+I_{i}-I_{s}, t\right) \\
& +\lambda_{0 i}^{+} u\left(k_{i}\right) r_{0 i}\left(k-I_{i}, t\right)-\left(\mu_{i} p_{i 0}+\mu_{i} p_{i j}^{-}\left(1-u\left(k_{j}\right)\right)\right) R_{i 0}\left(k+I_{i}, t\right) \\
& -\sum_{s=1}^{n}\left(\mu_{i} p_{i s}^{+}+\lambda_{0 i}^{(c)} q_{i s}\right) u\left(k_{s}\right) r_{i s}\left(\vec{k}+I_{i}-I_{s}, t\right) \\
& -\sum_{\substack{j, c=1 \\
j, c \neq i}}^{n} \mu_{i} p_{i s}^{-} q_{s c} u\left(k_{c}\right) r_{j i c}\left(\vec{k}+I_{j}+I_{i}-I_{c}, t\right) \\
& -R_{i}\left(\vec{k}+m I_{i}, t\right) \lambda_{0 i}^{(c)} q_{i 0} \sum_{m=1}^{\infty} \pi_{i m} \\
& -\lambda_{0 i}^{(c)} q_{i 0}\left(1-u\left(k_{i}\right)\right) \sum_{m=1}^{\infty} \pi_{i m} \sum_{r=1}^{m-1} R_{i}\left(\vec{k}+r I_{i}, t\right)-\mu_{i} p_{i s}^{-} q_{i s} \sum_{m=1}^{\infty} \pi_{s m} \\
& \left.\times R_{j i}\left(\vec{k}+I_{j}+m I_{i}, t\right)+\mu_{i} p_{i s}^{-}\left(1-u\left(k_{s}\right)\right) q_{s 0} \sum_{m=1}^{\infty} \pi_{s m} \sum_{r=1}^{m-1} R_{j i}\left(\vec{k}+I_{j}+r I_{i}, t\right)\right\} \\
& -\sum_{\substack{j, s=1 \\
j, s \neq i}}^{n} \mu_{i} p_{i s}^{-} q_{s c} u\left(k_{c}\right) r_{j s i}\left(\vec{k}+I_{j}+I_{i}-I_{c}, t\right)-\mu_{j} p_{j s}^{-} q_{s 0} \\
& \times \sum_{m=1}^{\infty} \pi_{s m} r_{i s}\left(\vec{k}+I_{i}+m I_{s}, t\right) \\
& +\mu_{j} p_{j s}^{-}\left(1-u\left(k_{s}\right)\right) q_{s 0} \sum_{m=1}^{\infty} \pi_{s m} \sum_{r=1}^{m-1} r_{i s}\left(\vec{k}+I_{i}+r I_{s}, t\right) .
\end{aligned}
$$


Let $\vec{V}^{T}(\vec{k}, t)=\left(v_{1}(\vec{k}, t), v_{2}(\vec{k}, t), \ldots, v_{n}(\vec{k}, t)\right)$. Then $(1)$ is representable in the form

$$
\begin{aligned}
\frac{d \vec{V}(\vec{k}, t)}{d t}= & -\Delta(\vec{k}) V(\vec{k}, t)+\sum_{i=0}^{n} \sum_{m=1}^{\infty} \sum_{r=-1}^{m} \Theta_{i j r m}(\vec{k}) \vec{V}\left(\vec{k}+I_{i}+r I_{j}, t\right) \\
& +\sum_{i, j, s=0}^{n} \Phi_{i j s}(\vec{k}) \vec{V}\left(\vec{k}+I_{i}+I_{j}-I_{s}, t\right)+\vec{E}(\vec{k}),
\end{aligned}
$$

where

$$
\begin{aligned}
& \vec{E}^{T}(\vec{k})=\left(E_{1}(\vec{k}), E_{2}(\vec{k}), \ldots, E_{n}(\vec{k})\right), \\
& E_{i}(\vec{k})=r_{i}(\vec{k})+\lambda_{0 i}^{+} u\left(k_{i}\right) r_{0 i}\left(k-I_{i}, t\right)-\left(\mu_{i} p_{i 0}+\mu_{i} p_{i j}^{-}\left(1-u\left(k_{j}\right)\right)\right) R_{i 0}\left(k+I_{i}, t\right) \\
& -\left(\mu_{i} p_{i s}^{+}+\lambda_{0 i}^{(c)} q_{i s}\right) u\left(k_{s}\right) r_{i s}\left(\vec{k}+I_{i}-I_{s}, t\right) \\
& -\mu_{i} p_{i s}^{-} q_{s c} u\left(k_{c}\right) r_{j i c}\left(\vec{k}+I_{j}+I_{i}-I_{c}, t\right)-\lambda_{0 i}^{(c)} q_{i 0} \sum_{m=1}^{\infty} \pi_{i m} R_{i}\left(\vec{k}+m I_{i}, t\right) \\
& -\lambda_{0 i}^{(c)} q_{i 0}\left(1-u\left(k_{i}\right)\right) \sum_{m=1}^{\infty} \pi_{i m} \sum_{r=1}^{m-1} R_{i}\left(\vec{k}+r I_{i}, t\right) \\
& -\mu_{i} p_{i s}^{-} q_{s 0} \sum_{m=1}^{\infty} \pi_{s m} R_{j i}\left(\vec{k}+I_{j}+m I_{i}, t\right) \\
& +\mu_{i} p_{i s}^{-}\left(1-u\left(k_{s}\right)\right) q_{s 0} \sum_{m=1}^{\infty} \pi_{s m} \sum_{r=1}^{m-1} R_{j i}\left(\vec{k}+I_{j}+r I_{i}, t\right) \\
& +\mu_{j} p_{j s}^{-}\left(1-u\left(k_{s}\right)\right) q_{s 0} \sum_{m=1}^{\infty} \pi_{s m} \sum_{r=1}^{m-1} r_{i s}\left(\vec{k}+I_{i}+r I_{s}, t\right) \\
& -\sum_{\substack{j, s=1 \\
j, s \neq i}}^{n} \mu_{i} p_{i s}^{-} q_{s c} u\left(k_{c}\right) r_{j s i}\left(\vec{k}+I_{j}+I_{i}-I_{c}, t\right) \\
& -\mu_{j} p_{j s}^{-} q_{s 0} \sum_{m=1}^{\infty} \pi_{s m} r_{i s}\left(\vec{k}+I_{i}+m I_{s}, t\right), \Phi_{i j s}(\vec{k})=\mu_{i} p_{i j}^{-} q_{j s} u\left(k_{s}\right), \\
& \Theta_{i j r m}(\vec{k})=\delta_{r-1} \delta_{m 1}\left\{\lambda_{0 i}^{+}+\left(\lambda_{0 j}^{+}+\mu_{j} p_{j i}^{+} u\left(k_{i}\right)-\mu_{i} p_{i j}^{+} u\left(k_{i}\right)\right)\left(1-\delta_{i j}\right)+\mu_{j} p_{j 0} u\left(k_{j}\right)\right. \\
& \left.+\sum_{s=1}^{n}\left(1-\delta_{s i}\right)\left(\mu_{i} p_{i 0}+\mu_{i} p_{i s}^{-}\left(1-u\left(k_{s}\right)\right)\right)\right\} \\
& +\delta_{r m}\left\{\sum_{j=1}^{n}\left(1-\delta_{i j}\right) \lambda_{0 j}^{(c)} q_{j 0} \pi_{j m}+\sum_{s=1}^{n}\left(1-\delta_{j s}\right) \mu_{j} p_{j s}^{-} q_{s 0} \pi_{s m}\right\} \\
& +\left(1-\delta_{r m}\right)(u(m+1)) \mu_{i} p_{i s}^{-}\left(1-u\left(k_{s}\right)\right) q_{s 0} \pi_{s m}+u(m) \mu_{j} p_{j s}^{-}\left(1-u\left(k_{s}\right)\right) q_{s 0} \pi_{s m} .
\end{aligned}
$$

$\delta_{i j}-$ the Kronecker symbol. 
Let us investigate the series $\sum_{i=1}^{n} \sum_{m=1}^{\infty} \sum_{r=-1}^{m} \Theta_{i j r m}$ for convergence. We get

$$
\begin{aligned}
\sum_{i=1}^{n} & \sum_{m=1}^{\infty} \sum_{r=1}^{m-1} \Theta_{i j r m} \\
= & \sum_{i=1}^{n} \sum_{m=1}^{\infty} \sum_{r=-1}^{m-1} \delta_{r-1} \delta_{m 1}\left\{\lambda_{0 i}^{+}+\left(\lambda_{0 j}^{+}+\mu_{j} p_{j i}^{+} u\left(k_{i}\right)-\mu_{i} p_{i j}^{+} u\left(k_{i}\right)\right)\left(1-\delta_{i j}\right)\right. \\
& \left.+\mu_{j} p_{j 0} u\left(k_{j}\right)+\sum_{s=1}^{n}\left(1-\delta_{s i}\right)\left(\mu_{i} p_{i 0}+\mu_{i} p_{i s}^{-}\left(1-u\left(k_{s}\right)\right)\right)\right\} \\
& +\delta_{r m}\left\{\sum_{j=1}^{n}\left(1-\delta_{i j}\right) \lambda_{0 j}^{(c)} q_{j 0} \pi_{j m}+\sum_{s=1}^{n}\left(1-\delta_{j s}\right) \mu_{j} p_{j s}^{-} q_{s 0} \pi_{s m}\right\} \\
& \left.+\left(1-\delta_{r m}\right)(u(r+1)) \mu_{i} p_{i s}^{-}\left(1-u\left(k_{s}\right)\right) q_{s 0} \pi_{s m}+u(r) \mu_{j} p_{j s}^{-}\left(1-u\left(k_{s}\right)\right) q_{s 0} \pi_{s m}\right) \\
= & \sum_{i, j=1}^{n} \lambda_{0 i}^{+}+\left(\lambda_{0 j}^{+}+\mu_{j} p_{j i}^{+} u\left(k_{i}\right)-\mu_{i} p_{i j}^{+} u\left(k_{i}\right)\right)\left(1-\delta_{i j}\right)+\mu_{j} p_{j 0} u\left(k_{j}\right) \\
& +\sum_{s=1}^{n}\left(1-\delta_{s i}\right)\left(\mu_{i} p_{i 0}+\mu_{i} p_{i s}^{-}\left(1-u\left(k_{s}\right)\right)\right) \\
& +\sum_{i=1}^{n} \sum_{j=1}^{n}\left(1-\delta_{i j}\right) \lambda_{0 j}^{(c)} q_{j 0} \sum_{m=1}^{\infty} \pi_{j m}+\sum_{s=1}^{n}\left(1-\delta_{j s}\right) \mu_{j} p_{j s}^{-} q_{s 0} \sum_{m=1}^{\infty} \pi_{s m} \\
& +\sum_{i=1}^{n} \mu_{i} p_{i s}^{-}\left(1-u\left(k_{s}\right)\right) \sum_{m=0}^{\infty} \pi_{s m} \sum_{r=1}^{m-1} 1
\end{aligned}
$$

From a practical point of view, we can assume that $\sum_{m=1}^{\infty} \pi_{i m}=1$, because a signal acting as a negative customer always destroys some non-zero number of positive customers. Therefore, the last expression is equal to

$$
\begin{aligned}
\sum_{i, j=1}^{n} \lambda_{0 i}^{+}+ & \left.\lambda_{0 j}^{+}+\mu_{j} p_{j i}^{+} u\left(k_{i}\right)-\mu_{i} p_{i j}^{+} u\left(k_{i}\right)\right)\left(1-\delta_{i j}\right)+\mu_{j} p_{j 0} u\left(k_{j}\right) \\
& +\sum_{s=1}^{n}\left(1-\delta_{s i}\right)\left(\mu_{i} p_{i 0}+\mu_{i} p_{i s}^{-}\left(1-u\left(k_{s}\right)\right)\right) \\
& +\sum_{i=1}^{n} \sum_{j=1}^{n}\left(1-\delta_{i j}\right) \lambda_{0 j}^{(c)} q_{j 0}+\sum_{s=1}^{n}\left(1-\delta_{j s}\right) \mu_{j} p_{j s}^{-} q_{s 0} \\
& +\sum_{i=1}^{n} \mu_{i} p_{i s}^{-}\left(1-u\left(k_{s}\right)\right) \sum_{m=0}^{\infty}(m-1) \pi_{s m} \\
& +\sum_{i=1}^{n} \mu_{j} p_{j s}^{-}\left(1-u\left(k_{s}\right)\right) q_{s 0} \sum_{m=1}^{\infty}(m+1) \pi_{s m}
\end{aligned}
$$




$$
\begin{aligned}
= & \sum_{i, j=1}^{n} \lambda_{0 i}^{+}+\left(\lambda_{0 j}^{+}+\mu_{j} p_{j i}^{+} u\left(k_{i}\right)-\mu_{i} p_{i j}^{+} u\left(k_{i}\right)\right)\left(1-\delta_{i j}\right)+\mu_{j} p_{j 0} u\left(k_{j}\right) \\
& +\sum_{s=1}^{n}\left(1-\delta_{s i}\right)\left(\mu_{i} p_{i 0}+\mu_{i} p_{i s}^{-}\left(1-u\left(k_{s}\right)\right)\right) \\
& +\sum_{i=1}^{n} \sum_{j=1}^{n}\left(1-\delta_{i j}\right) \lambda_{0 j}^{(c)} q_{j 0}+\sum_{s=1}^{n}\left(1-\delta_{j s}\right) \mu_{j} p_{j s}^{-} q_{s 0} \\
& +\sum_{i=1}^{n} \mu_{i} p_{i s}^{-}\left(1-u\left(k_{s}\right)\right)\left(b_{i}-1\right)+\sum_{i=1}^{n} \mu_{j} p_{j s}^{-}\left(1-u\left(k_{s}\right)\right) q_{s 0}\left(b_{i}+1\right) .
\end{aligned}
$$

This implies the convergence of the series.

It follows from (2) that

$$
\begin{aligned}
\vec{V}(\vec{k}, t)= & e^{-\Delta(\vec{k}) t}(\vec{V}(\vec{k}, 0)) \\
& +\int_{0}^{t} e^{\Delta(\vec{k}) x}\left\{\sum_{i, j=0}^{n} \sum_{m=1}^{\infty} \sum_{r=-1}^{m} \Theta_{i j r m}(\vec{k}) \vec{V}\left(\vec{k}+I_{i}+r I_{j}, t\right)\right. \\
& \left.\quad+\sum_{i, j, s=0}^{n} \Phi_{i j s}(\vec{k}) \vec{V}\left(\vec{k}+I_{i}+I_{j}-I_{s}, t\right)\right\} d x \\
+ & \frac{1}{\Delta(\vec{k})} \vec{E}(\vec{k})\left[1-e^{-\Delta(\vec{k}) t}\right] .
\end{aligned}
$$

Let $\vec{V}_{q}(\vec{k}, t)$ be approximation of $\vec{V}(\vec{k}, t)$ at $q$ th iteration, $\vec{V}_{q+1}(\vec{k}, t)$ - solution of (2) obtained by successive approximations. Then it follows from (3)

$$
\begin{aligned}
\vec{V}_{q+1}(\vec{k}, t)= & e^{-\Delta(\vec{k}) t}\left(\vec{V}_{q}(\vec{k}, 0)\right)+\int_{0}^{t} e^{\Delta(\vec{k}) x}\left\{\sum_{i, j=0}^{n} \sum_{m=1}^{\infty} \sum_{r=-1}^{m} \Theta_{i j r m}(\vec{k}) \vec{V}_{q}\left(\vec{k}+I_{i}+r I_{j}, t\right)\right. \\
& \left.+\sum_{i, j, s=0}^{n} \Phi_{i j s}(\vec{k}) \vec{V}_{q}\left(\vec{k}+I_{i}+I_{j}-I_{s}, t\right)\right\} d x+\frac{1}{\Delta(\vec{k})} \vec{E}(\vec{k})\left[1-e^{-\Delta(\vec{k}) t}\right] .
\end{aligned}
$$

As an initial approximation, we take the stationary distribution $\vec{V}_{0}(\vec{k}, t)=V(\vec{k})=$ $\lim _{t \rightarrow \infty} \vec{V}(\vec{k}, t)$, which satisfies the relation

$$
\begin{aligned}
\Delta(\vec{k}) \vec{V}(\vec{k})= & \sum_{i, j=1}^{n} \sum_{m=1}^{\infty} \sum_{r=-1}^{m} \Theta_{i j r m}(k) \vec{V}\left(k+I_{i}+r I_{j}\right) \\
& +\sum_{i, j, s=1}^{n} \Phi_{i j s}(k) \vec{V}\left(k+I_{i}+I_{j}-I_{s}\right)+\vec{E}(\vec{k})
\end{aligned}
$$

The following theorems are valid for successive approximations.

Theorem 1: Sequential approximations $\vec{V}_{q}(\vec{k}, t), q=0,1,2, \ldots$, converge for $t \rightarrow \infty$ to a stationary solution of the system of equations (2). 
Proof: Proof, as before, we shall carry out the method of mathematical induction. For the first approximation we have:

$$
\begin{aligned}
& \vec{V}_{1}(\vec{k}, t)=e^{-\Delta(\vec{k}) t}\left(\vec{V}_{0}(\vec{k}, 0)\right)+\int_{0}^{t} e^{\Delta(\vec{k}) x}\left\{\sum_{i, j=0}^{n} \sum_{m=1}^{\infty} \sum_{r=-1}^{m} \Theta_{i j r m}(\vec{k}) \vec{V}_{0}\left(\vec{k}+I_{i}+r I_{j}, t\right)\right. \\
& \left.+\sum_{i, j, s=0}^{n} \Phi_{i j s}(\vec{k}) \vec{V}_{0}\left(\vec{k}+I_{i}+I_{j}-I_{s}, t\right)\right\} d x+\frac{1}{\Delta(\vec{k})} \vec{E}(\vec{k})\left[1-e^{-\Delta(\vec{k}) t}\right] \\
& =e^{-\Delta(\vec{k}) t}(\vec{V}(\vec{k}, 0))+\int_{0}^{t} e^{\Delta(\vec{k}) x}\left\{\sum_{i, j=0}^{n} \sum_{m=1}^{\infty} \sum_{r=-1}^{m} \Theta_{i j r m}(\vec{k}) \vec{V}\left(\vec{k}+I_{i}+r I_{j}\right)\right. \\
& \left.+\sum_{i, j, s=0}^{n} \Phi_{i j s}(\vec{k}) \vec{V}\left(\vec{k}+I_{i}+I_{j}-I_{s}\right)\right\} d x+\frac{1}{\Delta(\vec{k})} \vec{E}(\vec{k})\left[1-e^{-\Delta(\vec{k}) t}\right] \\
& =e^{-\Delta(\vec{k}) t} \vec{V}(\vec{k}, 0)+\frac{1}{\Delta(\vec{k})}\left(1-e^{-\Delta(\vec{k}) t}\right) \\
& \times\left\{\sum_{i, j=0}^{n} \sum_{m=1}^{\infty} \sum_{r=-1}^{m} \Theta_{i j r m}(\vec{k}) \vec{V}\left(\vec{k}+I_{i}+r I_{j}\right)+\sum_{i, j, s=0}^{n} \Phi_{i j s}(\vec{k}) \vec{V}\left(\vec{k}+I_{i}+I_{j}-I_{s}\right)\right\} \\
& +\frac{1}{\Delta(\vec{k})} \vec{E}(\vec{k})\left[1-e^{-\Delta(\vec{k}) t}\right] \underset{t \rightarrow \infty}{\longrightarrow} \frac{1}{\Delta(\vec{k})} \\
& \times\left\{\sum_{i, j=0}^{n} \sum_{m=1}^{\infty} \sum_{r=-1}^{m} \Theta_{i j r m}(\vec{k}) \vec{V}\left(\vec{k}+I_{i}+r I_{j}\right)+\sum_{i, j, s=0}^{n} \Phi_{i j s}(\vec{k}) \vec{V}\left(\vec{k}+I_{i}+I_{j}-I_{s}\right)\right\} \\
& +\frac{1}{\Delta(\vec{k})} \vec{E}(\vec{k}) \text {. }
\end{aligned}
$$

From this it follows that for $q=1$ the theorem is satisfied. Suppose that the statement of the theorem is valid up to the $q$ th iteration. Then, using (4), and the L'Hospital rule, we have:

$$
\begin{aligned}
\lim _{t \rightarrow \infty} \vec{V}_{q+1}(\vec{k}, t)= & \lim _{t \rightarrow \infty} \frac{\int_{0}^{t} e^{\Delta(\vec{k}) x} \sum_{i=1}^{n} \sum_{m=1}^{\infty} \sum_{r=-1}^{m} \Theta_{i j r m}(k) \vec{V}_{q}\left(\vec{k}+r I_{i}+m I_{j}, x\right)}{e^{\Delta(\vec{k}) t}} \\
& +\lim _{t \rightarrow \infty} \frac{\int_{0}^{t} e^{\Delta(\vec{k}) x} \sum_{i, j, s=1}^{n} \Phi_{i j s}(k) \vec{V}_{q}\left(\vec{k}+I_{i}+I_{j}-I_{s}, x\right)}{e^{\Delta(\vec{k}) t}}+\frac{\vec{E}(\vec{k})}{\Delta(\vec{k})} \\
= & \lim _{t \rightarrow \infty} \frac{e^{\Delta(\vec{k}) t} \sum_{i=1}^{n} \sum_{m=1}^{\infty} \sum_{r=-1}^{m} \Theta_{i j r m}(k) \vec{V}_{q}\left(\vec{k}+r I_{i}+m I_{j}, t\right)}{\Delta(\vec{k}) e^{\Delta(\vec{k}) t}} \\
& +\lim _{t \rightarrow \infty} \frac{e^{\Delta(\vec{k}) t \sum_{i, j, s=1}^{n} \Phi_{i j s}(\vec{k}) \vec{V}_{q}\left(\vec{k}+I_{i}+I_{j}-I_{s}, t\right)}}{\Delta(\vec{k}) e^{\Delta(\vec{k}) t}}+\frac{\vec{E}(\vec{k})}{\Delta(\vec{k})}
\end{aligned}
$$




$$
\begin{aligned}
= & \sum_{i, j=1}^{n} \sum_{m=1}^{\infty} \sum_{r=-1}^{m} \Theta_{i j r m}(\vec{k}) \vec{V}\left(\vec{k}+I_{i}+r I_{j}\right) \\
& +\sum_{i, j, s=1}^{n} \Phi_{i j s}(\vec{k}) \vec{V}\left(\vec{k}+I_{i}+I_{j}-I_{s}\right)+\vec{E}(\vec{k})=\Delta(\vec{k}) \vec{V}(\vec{k}) .
\end{aligned}
$$

Therefore, the theorem is also true for $q+1$. Then, using the method of mathematical induction, we obtain the assertion of the theorem.

THEOREM 2: The sequence constructed according to scheme (4), for any zeroth approximation bounded in $\vec{V}_{0}(\vec{k}, t)$, converges for $q \rightarrow \infty$ to a unique solution of the system of equations (9).

Proof: Because $\vec{V}_{0}(\vec{k}, t)$ is limited function of $t$, then by $(4) \vec{V}_{1}(\vec{k}, t)$ is also limited, so

$$
\left|\vec{V}_{1}(\vec{k}, t)-\vec{V}_{0}(\vec{k}, t)\right| \leq C(\vec{k})
$$

Let us show that the inequality

$$
\left|\vec{V}_{q}(\vec{k}, t)-\vec{V}_{q-1}(\vec{k}, t)\right| \leq C^{*}\left(\eta_{1}^{*}+\varphi_{2}^{*}\right)^{q-1} \frac{t^{q-1}}{(q-1) !}
$$

where

$$
\max _{\vec{k}} \eta_{1}(\vec{k})=\eta_{1}^{*}, \quad \max _{\vec{k}} \varphi_{2}(\vec{k})=\varphi_{2}^{*}, \quad \max _{\vec{k}} c(\vec{k})=c^{*}
$$

As was proved earlier, the series $\eta_{1}(\vec{k})$ converges. It follows from $(6)$ that for $q=1$, this inequality holds. Suppose that it holds for $q=N$, and we show, using (4), its validity for $q=N+1$. We have:

$$
\begin{aligned}
& \left|\vec{V}_{N+1}(\vec{k}, t)-\vec{V}_{N}(\vec{k}, t)\right| \\
& =\mid e^{-\Delta(\vec{k}) t} \int_{0}^{t} e^{\Delta(\vec{k}) x}\left(\sum_{i, j=1}^{n} \sum_{m=1}^{\infty} \sum_{r=-1}^{m} \Theta_{i j m r}(\vec{k}) \vec{V}_{N}\left(\vec{k}+I_{i}+r I_{j}, x\right)\right. \\
& \left.\quad+\sum_{i, j, s=1}^{n} \Phi_{i j s}(\vec{k}) \vec{V}_{N}\left(\vec{k}+I_{i}+I_{j}-I_{s}, x\right)\right) d x+\frac{1}{\Delta(\vec{k})} \vec{E}(\vec{k})\left[1-e^{-\Delta(\vec{k}) t}\right] \\
& \quad-e^{-\Delta(\vec{k}) t} \int_{0}^{t} e^{\Delta(\vec{k}) x}\left(\sum_{i, j=1}^{n} \sum_{m=1}^{\infty} \sum_{r=-1}^{m} \Theta_{i j m r}(\vec{k}) \vec{V}_{N-1}\left(\vec{k}+I_{i}+r I_{j}, x\right)\right. \\
& \left.\quad+\sum_{i, j, s=1}^{n} \Phi_{i j s}(\vec{k}) \vec{V}_{N}\left(\vec{k}+I_{i}+I_{j}-I_{s}, x\right)\right) d x-\frac{1}{\Delta(\vec{k})} \vec{E}(\vec{k})\left[1-e^{-\Delta(\vec{k}) t}\right] \mid
\end{aligned}
$$




$$
\begin{aligned}
\leq & \mid e^{-\Delta(\vec{k}) t} \int_{0}^{t} e^{\Delta(\vec{k}) x}\left(\sum_{i, j=1}^{n} \sum_{m=1}^{\infty} \sum_{r=-1}^{m} \Theta_{i j m r}(\vec{k})\left(\vec{V}_{N}\left(\vec{k}+I_{i}+r I_{j}, x\right)-\vec{V}_{N-1}\left(\vec{k}+I_{i}+r I_{j}, x\right)\right)\right. \\
& \left.+\sum_{i, j, s=1}^{n} \Phi_{i j s}(\vec{k})\left(\vec{V}_{N}\left(\vec{k}+I_{i}+I_{j}-I_{s}, x\right)-\vec{V}_{N-1}\left(\vec{k}+I_{i}+I_{j}-I_{s}, x\right)\right)\right) d x \mid \\
\leq & e^{-\Delta(\vec{k}) t} \int_{0}^{t} e^{\Delta(\vec{k}) x}\left[\sum_{i, j=1}^{n} \sum_{m=1}^{\infty} \sum_{r=-1}^{m} \Theta_{i j m r}(\vec{k})\left|\vec{V}_{N}\left(\vec{k}+I_{i}+r I_{j}, x\right)-\vec{V}_{N-1}\left(\vec{k}+I_{i}+r I_{j}, x\right)\right|\right. \\
& \left.+\sum_{i, j, s=1}^{n} \Phi_{i j s}(\vec{k})\left|\vec{V}_{N}\left(\vec{k}+I_{i}+I_{j}-I_{s}, x\right)-\vec{V}_{N-1}\left(\vec{k}+I_{i}+I_{j}-I_{s}, x\right)\right|\right] d x \\
\leq & C^{*} e^{-\Delta(\vec{k}) t} \int_{0}^{t} e^{\Delta(\vec{k}) x}\left[\eta_{1}^{*}\left(\eta_{1}^{*}+\phi_{2}^{*}\right)^{N-1}+\phi_{2}^{*}\left(\eta_{1}^{*}+\phi_{2}^{*}\right)^{N-1}\right] \frac{x^{N-1}}{(N-1) !} d x \\
= & C^{*}\left(\eta_{1}^{*}+\varphi_{2}^{*}\right)^{N} e^{-\Delta(\vec{k}) t} \int_{0}^{t} e^{\Delta(\vec{k}) x} \frac{x^{N-1}}{(N-1) !} d x .
\end{aligned}
$$

From the inequality $e^{-\Lambda(\vec{k}) t} e^{\Lambda(\vec{k}) x} \leq 1, x \in[0, t]$, it follows that

$$
e^{-\Delta(\vec{k}) t} \int_{0}^{t} e^{\Delta(\vec{k}) x} \frac{x^{N-1}}{(N-1) !} d x \leq \int_{0}^{t} \frac{x^{N-1}}{(N-1) !} d x=\frac{t^{N}}{N !}
$$

therefore from (9) we obtain that inequality (7) takes place.

Because the

$$
\begin{aligned}
\lim _{q \rightarrow \infty} \vec{V}_{q}(\vec{k}, t) & =\lim _{q \rightarrow \infty}\left(\vec{V}_{0}(\vec{k}, t)+\sum_{n=0}^{m-1}\left(\vec{V}_{q+1}(\vec{k}, t)-\vec{V}_{q}(\vec{k}, t)\right)\right) \\
& =\vec{V}_{0}(\vec{k}, t)+\sum_{q=0}^{\infty}\left(\vec{V}_{q+1}(\vec{k}, t)-\vec{V}_{q}(\vec{k}, t)\right) \\
& \leq \vec{V}_{0}(k, t)+C^{*} \sum_{q=0}^{\infty} \frac{\left(\eta_{1}^{*} t+\phi_{2}^{*} t\right)^{q}}{q !}=\vec{V}_{0}(k, t)+C^{*} e^{\left(\eta_{1}^{*}+\phi_{2}^{*}\right) t}
\end{aligned}
$$

that is, limit of sequence $\left\{\vec{V}_{q}(\vec{k}, t)\right\}, q=0,1,2, \ldots$, exists, we denote it by $\vec{V}_{\infty}(\vec{k}, t)$. Substituting $\vec{V}_{\infty}(\vec{k}, t)$ into (3) instead of $\vec{V}(\vec{k}, t)$, we see that $\vec{V}_{\infty}(\vec{k}, t)$ is a solution of the system of equations (2) satisfying the initial conditions $\vec{V}_{\infty}(\vec{k}, 0)=\vec{V}(\vec{k}, 0)$ according to the previous theorem.

Suppose that there is another solution of the system of equations $(2) \vec{V}^{*}(\vec{k}, t)$. Then for it the relation (3) is valid if it is replaced in it $\vec{V}(\vec{k}, t), \vec{V}\left(\vec{k}+r I_{i}, t\right), \vec{V}\left(\vec{k}+I_{i}+I_{j}-I_{s}, t\right)$ respectively on $\vec{V}^{*}(\vec{k}, t), \vec{V}^{*}\left(\vec{k}+r I_{i}, t\right), \vec{V}^{*}\left(\vec{k}+I_{i}+I_{j}-I_{s}, t\right)$. Therefore, using (7), we have: 


$$
\begin{aligned}
& \left|\vec{V}_{q}(\vec{k}, t)-\vec{V}^{*}(\vec{k}, t)\right| \leq e^{-\Delta(\vec{k}) t}\left|\vec{V}_{q}(\vec{k}, t)-\vec{V}^{*}(\vec{k}, t)\right|+e^{-\Delta(\vec{k}) t} \int_{0}^{t} e^{-\Delta(\vec{k}) x} \\
& \quad \times\left[\left(\sum_{i=1}^{n} \sum_{m=1}^{\infty} \sum_{r=-1}^{m} \Theta_{i j m r}(\vec{k})\left|\vec{V}_{N}\left(\vec{k}+I_{i}+r I_{j}, x\right)-\vec{V}^{*}\left(\vec{k}+I_{i}+r I_{j}, x\right)\right|\right.\right. \\
& \left.\left.\quad+\sum_{i, j, s=1}^{n} \Phi_{i j s}(\vec{k})\left|\vec{V}_{N}\left(\vec{k}+I_{i}+I_{j}-I_{s}, x\right)-\vec{V}_{N-1}\left(\vec{k}+I_{i}+I_{j}-I_{s}, x\right)\right|\right)\right] d x .
\end{aligned}
$$

Similarly, as in the proof of inequality (7), it can be shown that inequality $\left|\vec{V}_{q}(\vec{k}, t)-\vec{V}^{*}(\vec{k}, t)\right| \leq M\left(\eta_{1}^{*}+\varphi_{2}^{*}\right)^{q}\left(t^{q} / q !\right)$, where $M$ is some constant. The right-hand side of this inequality tends to zero as the general term of the convergent series $\sum_{q=0}^{\infty} M\left(\eta_{1}^{*}+\varphi_{2}^{*}\right)^{q}\left(t^{q} / q !\right)=M e^{\left(\eta_{1}^{*}+\phi_{2}^{*}\right) t}$, therefore $\lim _{q \rightarrow \infty} \vec{V}_{q}(\vec{k}, t)=\vec{V}^{*}(\vec{k}, t)$. But we have already received earlier that $\lim _{q \rightarrow \infty} \vec{V}_{q}(\vec{k}, t)=\vec{V}(\vec{k}, t)$, so $\vec{V}^{*}(\vec{k}, t)=\vec{V}(\vec{k}, t)$, which proves the uniqueness of the solution of the system of equations (2).

THEOREM 3: Each successive approximation $\vec{V}_{q}(\vec{k}, t), q \geq 1$, is representable in the form of a convergent power series

$$
\vec{V}_{q}(\vec{k}, t)=\sum_{l=0}^{\infty} g_{q l}(\vec{k}) t^{l}
$$

whose coefficients satisfy the recurrence relations:

$$
\begin{aligned}
g_{q+1 l}(\vec{k}) & =\frac{-\Delta(\vec{k})^{l}}{l !}\left\{\vec{V}(\vec{k}, 0)-\frac{\vec{E}(\vec{k})}{\Delta(\vec{k})}+\sum_{u=0}^{l-1} \frac{(-1)^{u+1} u !}{\Delta(\vec{k})^{u+1}} G_{q l}(\vec{k})\right\}, l \geq 0, \\
g_{q 0}(\vec{k}) & =\vec{V}(\vec{k}, 0), g_{0 l}(\vec{k})=\vec{V}(\vec{k}, 0) \delta_{l 0}, \\
G_{q l}(\vec{k}) & =\sum_{i, j=1}^{n}\left[\sum_{m=1}^{\infty} \sum_{r=-1}^{m} \Theta_{i j m r}(\vec{k}) g_{q l}\left(\vec{k}+I_{i}+r I_{j}\right)+\sum_{s=1}^{n} \Phi_{i j s}(\vec{k}) g_{q l}\left(\vec{k}+I_{i}+I_{j}-I_{s}\right)\right] .
\end{aligned}
$$

PROOF: We show that the coefficients of the power series (10) satisfy the recurrence relations (11). We substitute the successive approximations (10) in relation (4). Then, given that

$$
e^{-\Delta(\vec{k}) t} \int_{0}^{t} e^{\Delta(\vec{k}) x} x^{l} d x=\left[\frac{1}{\Delta(\vec{k})}\right]^{l+1} l ! \sum_{j=l+1}^{\infty} \frac{[-\Delta(\vec{k})]^{l}}{j !}, \quad l=0,1,2, \ldots
$$

we obtain

$$
\begin{aligned}
\sum_{l=0}^{\infty} g_{q l}(\vec{k}) t^{l}= & e^{-\Delta(\vec{k}) t} \vec{V}(\vec{k}, 0)+\frac{\vec{E}(\vec{k})}{\Delta(\vec{k})}\left[1-e^{-\Delta(\vec{k}) t}\right] \\
& +\sum_{l=0}^{\infty} \sum_{i, j=1}^{n}\left[\sum_{m=1}^{\infty} \sum_{r=-1}^{m} \Theta_{i j r m}(\vec{k}) g_{q l}\left(\vec{k}+I_{i}+r I_{j}\right)+\sum_{s=1}^{n} \Phi_{i j s}(\vec{k}) g_{q l}\left(\vec{k}+I_{i}+I_{j}-I_{s}\right)\right] \\
& \times\left[\frac{1}{\Delta(\vec{k})}\right]^{l+1} l !+\sum_{u=l+1}^{\infty} \frac{[-\Delta(k)]^{u}}{u !} .
\end{aligned}
$$


Using (11), this series can be written in the form

$$
\begin{aligned}
\sum_{l=0}^{\infty} g_{q l}(k) t^{l}= & e^{-\Delta(\vec{k}) t} \vec{V}(\vec{k}, 0)-\frac{\vec{E}(\vec{k})}{\Delta(\vec{k})}\left[1-e^{-\Delta(\vec{k}) t}\right] \\
& +\sum_{l=0}^{\infty} G_{q l}(\vec{k})\left[\frac{1}{\Delta(\vec{k})}\right]^{l+1} l ! \sum_{u=l+1}^{\infty} \frac{[-\Delta(\vec{k})]^{u}}{u !} t^{u}
\end{aligned}
$$

After interchanging summation indices and expanding $e^{-\Delta(\vec{k}) t}$ in a series in powers of $t$, we have

$$
\sum_{l=0}^{\infty} g_{q l}(k) t^{l}=\frac{\vec{E}(\vec{k})}{\Delta(\vec{k})}+\sum_{l=0}^{\infty} \frac{[-\Delta(k)]^{l}}{l !}\left\{\vec{V}(\vec{k}, 0)-\frac{\vec{E}(\vec{k})}{\Delta(\vec{k})}+\sum_{u=0}^{l-1} \frac{(-1)^{u+1} u !}{[\Delta(\vec{k})]^{u+1}} G_{q u}(\vec{k})\right\} t^{l}
$$

If we equate the coefficients of $t^{l}$ in expression (12), we obtain the relations (11) for the coefficients of the series (10).

To find the radius of convergence of the power series (10), we can use the CauchyHadamard formula $\frac{1}{R_{1}(k)}=\lim _{l \rightarrow \infty} \sqrt[l]{\left|g_{q l}(k)\right|}$. Similarly, as in $[12,14]$, it can be shown that the radius of convergence of the series (10) is equal to $+\infty$.

\section{MODEL EXAMPLE}

Let us $n=7$ be seven systems be five QS. Let the probabilities of arrive of customers in the $i$ th system be equal, respectively $p_{0 i}^{+}=0,15, i=\overline{1,6}, p_{07}^{+}=0,1 ; \quad p_{0 i}^{-}=0,14, p_{06}^{-}=$ $p_{07}^{-}=0,15$, and $\sum_{i=1}^{7} p_{0 i}^{+}=1, \sum_{i=1}^{7} p_{0 i}^{-}=1$. Let also the intensities of incoming streams of positive and negative customers are equal, respectively $\lambda^{+}=100$ and $\lambda^{(c)}=90$.

Then the intensities of the input flow of positive customers and signals to each of the QS $\lambda_{0 i}^{+}$and $\lambda_{0 i}^{(c)}$, respectively will be equal to:

$$
\lambda_{0 i}^{+}=15, i=\overline{1,6}, \lambda_{07}^{+}=10, \quad \lambda_{0 i}^{(c)}=12,6, i=\overline{1,5}, \quad \lambda_{06}^{(c)}=\lambda_{07}^{(c)}=12,6 .
$$

Let us the customer service intensities in the network systems are equal: $\mu_{i}=50, i=\overline{1,5}$.

Suppose also that the probabilities of the transitions of positive and negative customers between the QS networks are equal

$$
\begin{aligned}
& p_{11}^{+}=0,1, p_{11}^{-}=0,1, p_{12}^{+}=0,1, p_{12}^{-}=0,1 \\
& p_{1 i}^{+}=0,05, p_{1 i}^{-}=0,05, i=\overline{3,7}, p_{10}=1-\sum_{j=1}^{5}\left(p_{1 j}^{+}+p_{1 j}^{-}\right)=0,1 \\
& p_{21}^{+}=0,1, p_{21}^{-}=0,1, p_{22}^{+}=0,1, p_{22}^{-}=0,1
\end{aligned}
$$




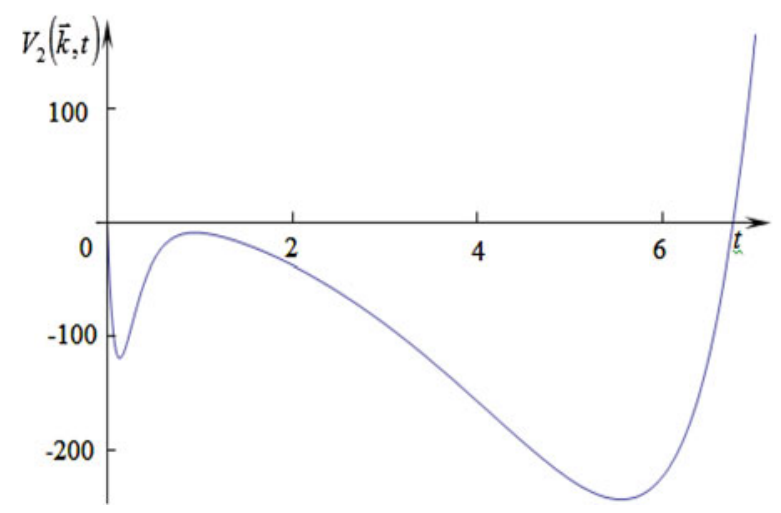

FIGURE 1. Revenues of the second QS in a period of time $[0 ; 7]$.

$$
\begin{aligned}
& p_{2 i}^{-}=0,05, p_{2 i}^{+}=0,05, i=\overline{3,7}, p_{j i}^{-}=0,05, p_{j i}^{+}=0,05, i=\overline{1,3}, \\
& p_{20}=1-\sum_{j=1}^{5}\left(p_{2 j}^{+}+p_{2 j}^{-}\right)=0,1, p_{j i}^{-}=0,05, p_{j i}^{+}=0,05, i=\overline{4,7}, j=\overline{3,5} \\
& p_{i 0}=1-\sum_{j=1}^{5}\left(p_{i j}^{+}+p_{i j}^{-}\right)=0,3, i=\overline{3,5} \cdot q_{i j}=0,15, i \neq j, i, j>0, q_{i i}=0, q_{i 0}=0,1 .
\end{aligned}
$$

Let us the random variable $B_{i}$ has a Poisson distribution with parameter $\lambda>0$ and $m=15$, then $\pi_{i m}=\left(\lambda^{m} / m !\right) e^{-\lambda}, m=\overline{0,5}, \quad i=\overline{1,5}$. Let us also $\lambda=5, r_{i s}(\vec{k})=$ $2000 \sin (\pi / 10) i ; r_{i j s}(\vec{k})=2000 e^{i+j+s} ; R_{i}(\vec{k})=100 ; R_{i j}(\vec{k})=500 . i, j, s=\overline{1,5}$.

Find the expected revenue of the first QS $v_{2}(\vec{k}, t)$ at $\vec{k}=(1,2,1,2,1,2,1)$ under the initial condition $v_{1}(\vec{k}, 0)=0$. Solving the problem using the programming language $\mathrm{C}$ \# on the interval $[0,7]$ with $\varepsilon=10^{-6}$, we obtain the dependence presented in Figure 1 .

The number of terms in the series was found using $\left|g_{q l}\left(\vec{k}^{*}\right)\right| \leq \varepsilon$, where $k^{*}: g_{q l}\left(\vec{k}^{*}\right)=$ $\max _{\vec{k}}\left|g_{q l}(\vec{k})\right|$, and the number of iterations $q$, using inequality $\mid \vec{V}_{q+1}(1,2,1,2,1,2,1 t)-$ $\vec{V}_{q}(1,2,1,2,1,2,1, t) \mid \leq \varepsilon$. We obtained that the number of iterations $q^{*}=50$, while the terms of the series $l^{*}=82$.

\section{CONCLUSIONS}

The paper investigates the Markov G-network with signals and revenues in the case when the signal can destroy a group of positive customers. For such a network, the expected revenues in the network systems were found using the method of successive approximations, combined with the method of series.

Further research in this direction may be associated with finding of variances of revenues of network systems.

\section{References}

1. Stidham, S. \& Weber, R. (1993). A survey of Markov decision models for control of networks of queues. Queueing Systems 13(1-3): 291-314. 
2. Matalytski, M. (2009). On some results in analysis and optimization in the Markov networks with incomes and their application. Automation and Remote Control 70(2): 1689-1697.

3. Matalytski, M. (2015). Analysis and forecasting of expected incomes in Markov networks with bounded waiting time for the claims. Automation and Remote Control 76(6): 1005-1017.

4. Matalytski, M. (2015). Analysis and forecasting of expected incomes in Markov networks with unreliable servicing systems. Automation and Remote Control 76(3): 2179-2189.

5. Matalytski, M. (2017). Forecasting anticipated income in the Markov networks with positive and negative customers. Automation and Remote Control 78(5): 815-825.

6. Gelenbe, E., Gellman, M., Lent, R., Liu, P., \& Su, P. (2004). Autonomous smart routing for network QoS. 1st International Conference on Autonomic Computing, Proceedings. International Conference, pp. 232-239.

7. Gelenbe, E. \& Iasnogorodsky, R. (1980). A queue with server of walking type. Annales de l'Institut Henri Poincaré, Section B: Calcul de Probabilités et Statistiques. 16(1): 63-73.

8. Gelenbe, E. \& Fourneau, J.-M. (2002). G-Networks with resets. Performance Evaluation 49(1-4): 179-191.

9. Gelenbe, E. (1991). Product form Queueing networks with negative and positive customers. Journal of Applied Probability 28: 656-663.

10. Gelenbe, E. (1993). G-networks with triggered customer movement. Journal of Applied Probability 30: $742-748$.

11. Gelenbe, E. (1993). G-networks with signals and batch removal. Probability in the Engineering and Informational Sciences 7: 335-342.

12. Matalytski, M. \& Naumenko, V. (2016). Stochastic networks with nonstandard moving customers. Monograph. Grodno: GrSU, 348 p.

13. Matalytski, M. (2008). Investigation Markov HM-networks with multiple class customers. News of NS RB, Series Physics and Mathematics Science 4(4): 113-119.

14. Kosareva, E.V., Matalytski, M.A., \& Rozov, K.V. (2012). About finding incomes in HM-networks with limited waiting time applications by successive approximations, combined with the method of series. VesnikHrodzenskahaDziarzhaunahaUniversitetaImiaIankiKupaly. Seryia 2. 3, 125-130. 